Editorial

\title{
FORMACIÓN DE ENFERMERÍA EN CUIDADOS PALIATIVOS
}

\section{PALLIATIVE CARE NURSING EDUCATION}

\author{
Aintzane Gallastegui Braña \\ Enfermera \\ Especialista en Cuidados Paliativos y Oncología, Diplomada en Bioética \\ Profesora Clínica Asistente, Escuela de Enfermería, Programa de Medicina Paliativa y \\ Cuidados Continuos, Pontificia Universidad Católica de Chile \\ agallastegui@uc.cl \\ http://dx.doi.org/0000-0002-9927-2801
}

http://dx.doi.org/10.7764/Horiz_Enferm.32.3.232-236

\section{INTRODUCCIÓN}

\section{La realidad del sufrimiento asociado a la enfermedad}

La pandemia del Sars-CoV-2 vino no solo a revolucionar nuestra forma de vivir, sino también de morir. Esta crisis sanitaria, cuyo costo humano posiblemente aún no logramos dimensionar, fue capaz de hacer visible el sufrimiento y la muerte vivida por quienes sucumbían ante este nuevo virus a toda la sociedad. Para el personal sanitario, esta situación lo enfrentó a la realidad de la muerte desnuda, pasando a estar a cargo no solo del cuidado físico de la persona, el más habitualmente abordado, sino también de su acompañamiento emocional, social y espiritual hasta su último respiro.

Enfermería cumple un rol fundamental a la hora de poder cuidar y acompañar a quienes viven con enfermedades avanzadas y especialmente al final de su vida, dado que es el profesional clínico que más tiempo pasa al lado de ellos ${ }^{(1-4)}$. Es así que no es de extrañar que, si nos remontamos a los orígenes de la enfermería, el cuidado de la persona sufriente y al final de su vida se encuentra enraizado en su esencia disciplinar ${ }^{(5)}$. La misma fundación de la enfermería moderna se basó en el cuidado de las personas más graves y cercanas a la muerte, mientras Florence Nightingale cuidaba de soldados malheridos y moribundos durante la guerra de Crimea $^{(6)}$. Esta acción se basaba en la filosofía del cuidado de las necesidades físicas, emocionales, espirituales y ambientales de la persona en fin de vida, permitiendo al paciente estar de la mejor manera para que la naturaleza actuara sobre él ${ }^{(7)}$.

De esta manera, los cuidados paliativos, disciplina encargada del cuidado activo y holístico de personas con sufrimiento grave debido a una enfermedad, y especialmente de quienes están cerca del final de la vida ${ }^{(8)}$, parecieran fundarse en los orígenes filosóficos del cuidado holístico de enfermería, de la mano de la trabajadora social, enfermera y médica Madame Cicely Saunders. Los cuidados paliativos son considerados una disciplina con un alto componente de enfermería dados estos elementos comunes y las necesidades específicas 
de la población que atiende. Es así como la evidencia demuestra que las enfermeras son quienes realizan el $70 \%$ de las intervenciones en este tipo de pacientes, cifra que aumenta al $85 \%$ en la atención domiciliaria, y en el $39 \%$ es la única profesional involucrada en su cuidado directo $^{(9)}$.

En la actualidad, la necesidad de contar con profesionales formados en cuidados paliativos, especialmente enfermeros y enfermeras, es un imperativo social y ético. El mundo vive otra pandemia que será de más largo aliento, la del problema del sufrimiento asociado a la enfermedad. El envejecimiento poblacional con el aumento en la prevalencia de las enfermedades crónicas no transmisibles ${ }^{(10-13)}$, que hoy causan el $85 \%$ de las muertes en Chile al año ${ }^{(14)}$, ha llevado a que si hace 100 años el periodo de discapacidad previo a la muerte era de pocos días a semanas (debido a enfermedades infecciosas), hoy ese periodo se ha extendido en promedio a dos años ${ }^{(15)}$. Esto se traduce no solo en aumento de la demanda de atenciones en los servicios de salud en forma prolongada, sino también en un impacto significativo en la calidad de vida de las personas enfermas, sus familias y cuidadores. Se estima que, al año, más de 60 millones de personas viven con sufrimiento asociado a la enfermedad, equivalentes a 6 billones de días de sufrimiento anuales, pero solo el $14 \%$ tiene acceso a cuidados adecuados para aliviarlo ${ }^{(16)}$.

De esta manera, la Organización Mundial de la Salud, ha declarado los cuidados paliativos como un derecho humano y parte fundamental del sistema integrado en salud de los países ${ }^{(17)}$. La falta de formación en cuidados paliativos del personal sanitario ha sido identificada internacionalmente como una barrera para garantizar el acceso a estos cuidados $^{(18)}$, por lo que diversas organizaciones internacionales plantean la formación universal en cuidados paliativos al menos básica en todos los futuros profesionales de la salud desde el pregrado como una de las estrategias centrales para abordar este problema ${ }^{(19-20)}$.

Enfermería tiene la oportunidad y el deber de liderar la respuesta para el alivio del sufrimiento asociado a la enfermedad. Como expertos y expertas del cuidado, jamás podremos decir "no hay nada más que hacer" a nuestros pacientes y familias, pues siempre podremos cuidar. Pero para ello, se hace indispensable la formación desde el pregrado en cuidados paliativos y la adquisición de competencias complejas como son las que se requieren para acompañar a personas con enfermedades graves y cerca del final de la vida. Las habilidades de comunicación, el pensamiento crítico, la identificación y análisis de problemas ético-clínicos, los cuidados de transición, el trabajo interdisciplinario, entre otros, son herramientas claves en este contexto ${ }^{(21-23)}$.

A su vez, estas habilidades constituyen competencias trasversales y deseables en todo futuro profesional del área de la salud. El cuidado de enfermería en el contexto paliativo exige un pensamiento crítico avanzado, con altos niveles de abstracción, reflexión y la puesta en marcha de habilidades complejas ${ }^{(24)}$ que permitirán no solo cuidar mejor a los pacientes con enfermedades avanzadas y sus familias, sino también formar profesionales más humanos, integrales y críticos de su quehacer, capaces de brindar cuidados compasivos a quienes tengan la oportunidad de cuidar en cualquier contexto de salud. Por otro lado, las experiencias internacionales de formación en cuidados paliativos en el pregrado de enfermería, demuestran la estrecha relación entre estas dos disciplinas. Los alumnos 
manifiestan que a través de la formación en cuidados paliativos fueron capaces de vivir y comprender mejor la esencia disciplinar de enfermería, asignándole un valor central en esta al acto de cuidar y reconociendo a la persona como el centro de ese cuidado ${ }^{(25-26)}$.

Ante la pronta entrada en vigencia en Chile de la Ley sobre el "Reconocimiento y protección de los derechos de las personas con enfermedades terminales y el buen morir", como disciplina tenemos la oportunidad de liderar su implementación, dar respuesta a las necesidades de una población con enfermedades causantes de sufrimiento en aumento, y a su vez, formar profesionales que vivan y transmitan el corazón de la enfermería, independiente de su lugar de desempeño futuro.

La experiencia del sufrimiento y la muerte está presente siempre en la historia de vida de aquellos a los que la enfermera cuida. Enfermería es testigo diario de esa realidad. Formar profesionales capaces de responder competente, compasiva, humana y holísticamente a esta, no es solo una necesidad, sino también, un deber. Porque, aunque el modelo biomédico afirme que "ya no hay nada más que hacer", enfermería siempre podrá cuidar.

\section{REFERENCIAS BIBLIOGRÁFICAS}

1. Smith MB, Macieira TGR, Bumbach MD, et al. The Use of Simulation to Teach Nursing Students and Clinicians Palliative Care and End-of-Life Communication: A Systematic Review. Am J Hosp Palliat Care. 2018; 35(8):1140-1154. doi:10.1177/1049909118761386

2. Khraisat OM, Hamdan M, Ghazzawwi M. Palliative Care Issues and Challenges in Saudi Arabia: Knowledge Assessment Among Nursing Students. J Palliat Care. 2017; 32(34):121-126. doi:10.1177/0825859717743229

3. Sopcheck J. Social, Economic, and Political Issues Affecting End-of-Life Care. Policy Polit Nurs Pract. 2016; 17(1):32-42. doi:10.1177/1527154416642664

4. Sarabia-Cobo CM, Alconero-Camarero AR, Lavín-Alconero L, Ibáñez-Rementería I. Assessment of a learning intervention in palliative care based on clinical simulations for nursing students. Nurse Educ Today. 2016; 45:219-224. doi:10.1016/j.nedt.2016.08.014

5. Lynch M, Dahlin C, Hultmand T, Coakley E. Palliative care nursing - defining the discipline? J Hospice Palliat Nurs. 2011; 13(2): 106-11.

6. Dahlin C, Lynch M. Evolution of the advanced practice nurse in palliative care. In: Dahlin CM, Lynch MT, eds. Core Curriculum for the advanced practice hospice and palliative registered nurse. 2nd ed. Pittsburg, PA: Hospice and Palliative Nurse Association; 2013:312.

7. Light KM. Florence Nightingale and holistic philosophy. J Holist Nurs. 1997;15(1):2540. doi:10.1177/089801019701500104

8. Radbruch L, De Lima L, Knaul F, et al. Redefining Palliative Care-A New ConsensusBased Definition. J Pain Symptom Manage. 2020; 60(4):754-764. doi:10.1016/j.jpainsymman.2020.04.027

9. Singer, A., Goebel, J., Kim, Y. et al. Populations and interventions for palliative and enfof-life care: A systematic review. J Palliat Med. 2016 Sep;19(9):995-1008 
10. World Health Organization. Death by cause. The Global Health Observatory; 2020 [Internet]. $\quad$ [Consultado $19 \quad$ Oct 2021$]. \quad$ Disponible en: who.int/data/gho/data/themes/mortality-and-global-health-estimates/ghe-leadingcauses-of-death

11. Jones DS, Podolsky SH, Greene JA. The burden of disease and the changing task of medicine. $N$ Engl J Med. 2012;366(25):2333-2338. doi:10.1056/NEJMp1113569

12. James SL, Abate D, Abate KH, et al. Global, regional, and national incidence, prevalence, and years lived with disability for 354 Diseases and Injuries for 195 countries and territories, 1990-2017: A systematic analysis for the Global Burden of Disease Study 2017. Lancet 2018; 392: 1789-1858.

13. World Health Organization. Noncommunicable diseases; 2021 [Internet]. [Consultado 19 Oct 2021]. Disponible en: https://www.who.int/en/news-room/factsheets/detail/noncommunicable-diseases

14. Instituto Nacional de Estadísticas (2021) Esperanza de Vida. Recuperado de: https://www.ine.cl/ine-ciudadano/definiciones-estadisticas/poblacion/esperanza-de-vida

15. Heinle R, McNulty J, Hebert RS. Nurse practitioners and the growth of palliative medicine. Am J Hosp Palliat Care. 2014;31(3):287-291. doi:10.1177/1049909113489163

16. Knaul FM, Farmer PE, Krakauer EL, et al. Alleviating the access abyss in palliative care and pain relief-an imperative of universal health coverage: the Lancet Commission report [published correction appears in Lancet. 2018 Mar 9;:]. Lancet. 2018;391(10128):13911454. doi:10.1016/S0140-6736(17)32513-8

17. World Health Organization. Fortalecimiento de los cuidados paliativos como parte del tratamiento integral a lo largo de la vida. Resolución de la 67 a Asamblea Mundial de la Salud, WHA67.19, 2014. Disponible en: http://apps.who.int/ medicinedocs/documents/s21454es/s21454es.pdf

18. Lynch T, Clark D, Centeno C, Rocafort J, de Lima L, Filbet M, et al. Barriers to the development of palliative care in Western Europe. Palliat Med 2010; 24: 812-819

19. World Health Organization. Strengthening of palliative care as a component of integrated treatment within the continuum of care. 134th sesión, Agenda Item 9.4 2014. Disponible en: http://apps.who.int/ medicinedocs/documents/s21454es/s21454es.pdf

20. Radbruch L, de Lima L, Lohmann D, Gwyther E, Payne S. The Prague Charter: urging governments to relieve suffering and ensure the right to palliative care. Palliat Med.2013; 27(2):101-102. doi:10.1177/0269216312473058

21. Anderson WG, Puntillo K, Cimino J, et al. Palliative care professional development for critical care nurses: a multicenter program. Am J Crit Care. 2017; 26(5):361-371.11.

22. Dudley N, Chapman S, Spetz J. Community-based palliative care leader perspectives on staffing, recruitment, and training. J Hosp Palliat Nurs. 2018;20(2):146-152.

23. Buller H, Virani R, Malloy P, Paice J. End-of-Life Nursing and Education Consortium Communication Curriculum for Nurses. J Hosp Palliat Nurs. 2019;21(2):E5-E12. doi:10.1097/NJH.0000000000000540

24. Bradley, E., Cherlin, E., McCorkle, R. et al. Nurses' use of palliative care practices in the acute care setting. J Prof Nurs 2001; 17:14-22. 
25. Ballesteros M, Centeno C, Arantzamendi M. A qualitative exploratory study of nursing students' assessment of the contribution of palliative care learning. Nurse Educ Today. 2014; 34(6):e1-e6. doi:10.1016/j.nedt.2013.12.010

26. Hold JL, Blake BJ, Ward EN. Perceptions and experiences of nursing students enrolled in a palliative and end-of-life nursing elective: A qualitative study. Nurse Educ Today. 2015; 35(6):777-781. doi:10.1016/j.nedt.2015.02.011 